\title{
CRRT: trattamento della crisi metabolica grave nella maple syrup urine disease
}

Giornale di Tecniche Nefrologiche e Dialitiche 2018, Vol. 30(I) 24-27

(C) The Author(s) 2018

Reprints and permissions:

sagepub.co.uk/journalsPermissions.nav

DOI: I0.1 I77/03949362। 8760828

journals.sagepub.com/home/gtn

\section{(SAGE}

\author{
Pasquale Fatuzzo, Rossella R Marchese, Roberta Aliotta, \\ Alessandra Pani e Luca Zanoli
}

\begin{abstract}
Maple syrup urine disease (MSUD) is a rare disorder of the amino acid metabolism that is transmitted as an autosomal recessive trait and is characterized by deficiency of the branched-chain $\alpha$-keto acid dehydrogenase enzyme complex (BCKDC). Reduced BCKDC activity results in increased plasma concentrations of leucine, isoleucine and valine. Leucine storage is mainly accountable for clinical symptoms within 48 hours of birth, more infrequently later in life. In subsequent years, patients with MSUD can develop acute metabolic decompensation, which may manifest as lethargy and/or coma, along with a high risk of cerebral edema and exitus. Sudden treatment of acute metabolic disorders is required: suppression of catabolism and utilization of amino acids in excess for protein synthesis and prompt removal of accumulated toxic metabolites by extracorporeal techniques (intermittent hemodialysis, continuous renal replacement therapies, peritoneal dialysis) or exchange transfusion. We discuss the case of a 14-year-old patient suffering from MSUD who was hospitalized for acute metabolic failure. The patient was at first treated only with nutritional support, and then with continuous veno-venous hemofiltration (CVVH) for severe neurological involvement.
\end{abstract}

\section{Keywords}

Acute metabolic failure, $\mathrm{CVVH}$, maple syrup urine disease

\section{Introduzione}

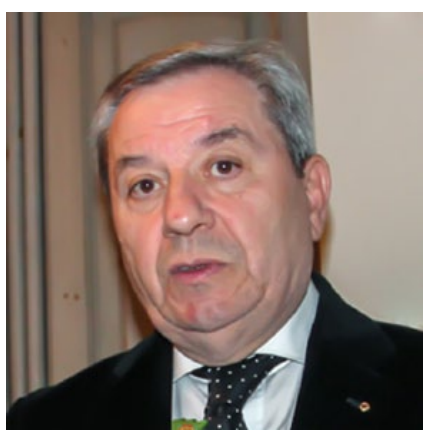

La malattia delle urine a sciroppo d'acero (MSUD), nota anche come leucinosi, è una rara aminoacidopatia trasmessa con modalità autosomica recessiva, caratterizzata dal deficit del complesso enzimatico $\alpha$-chetoacidodeidrogenasi a catena ramificata (BCKDC). ${ }^{1}$

Quest'ultimo è il secondo enzima della via metabolica degli aminoacidi a catena ramificata (BCAA) leucina, isoleucina e valina. I BCAA sono precursori necessari per la gluconeogenesi, per la produzione di energia e per la sintesi di acidi grassi e colesterolo. I prodotti terminali delle loro vie metaboliche sono acetil-coenzimaA (CoA), acetoacetato e succinil-CoA.
Il complesso enzimatico BCKDC, sito all'interno della membrana mitocondriale, catalizza la decarbossilazione degli $\alpha$-ketoacidi di leucina, isoleucina e valina nei loro rispettivi acil-CoA a catena ramificata. ${ }^{1}$ La ridotta attività del suddetto complesso enzimatico determina un incremento delle concentrazioni plasmatiche dei BCAA e dei loro corrispondenti chetoacidi.

La malattia è caratterizzata da 5 varianti fenotipiche: classica, intermittente, intermedia, tiamino-sensibile e da deficit di diidrolipoil-deidrogenasi (E3). Esordisce

Dipartmento di Medicina clinica e sperimentale, Nefrologia

Divisione, Policlinico Universitario, Universitá di Catilina, Catilina, Italia

\section{Corrispondenza:}

Pasquale Fatuzzo, MD., Dipartimento di Medicina Clinica e Sperimentale, Divisione di Nefrologia, Policlinico Universitario, Universita di Catania, Via Santa Sofia 78, 95123 Catania, Italia E-mail: fatuzzo@unict.it 
Tabella I. Le caratteristiche tecniche impiegate per il trattamento di CVVH. Abbiamo scelto di immettere la soluzione di sostituzione sia a valle che a monte del filtro (rispettivamente postdiluizione e prediluizione), in quanto, l'uso contemporaneo di pre- e postdiluizione è una soluzione che consente il mantenimento dell'efficienza del filtro e di una elevata clearance dei metaboliti a basso peso molecolare, come la leucina.

Caratteristiche tecniche trattamento $\mathrm{CVVH}$

\begin{tabular}{ll}
\hline Accesso vascolare & CVC temporaneo doppio lume v. femorale dx \\
Durata trattamento & 4320 minuti \\
Sistema & Aquarius con filtro Acquamax \\
Flusso ematico medio & $200 \mathrm{ml} / \mathrm{min}$ \\
Dose terapeutica & $40 \mathrm{ml} / \mathrm{kg} / \mathrm{h}(\mathrm{PC} 48 \mathrm{~kg})$ \\
Prediluizione & $1000 \mathrm{ml} / \mathrm{h}$ \\
Postdiluizione & $1000 \mathrm{ml} / \mathrm{h}$ \\
UF netta & $0 \mathrm{ml} / \mathrm{h}$ \\
Anticoagulazione & eparina sodica $12.5 \mathrm{Ul} / \mathrm{kg} / \mathrm{h} \mathrm{(tot.} 600 \mathrm{Ul} / \mathrm{h})$ \\
Soluzione dialisi & Accusol con KCL $3 \mathrm{mmol} / \mathrm{L}$
\end{tabular}

Tabella 2. Le concentrazioni plasmatiche dei BCAA prima del trattamento (giorno 0) durante il trattamento con CVVH (giorni I, 2 , 3) e nel post-trattamento (giorni da 4 a 8).

\begin{tabular}{llrr}
\hline Giorni & Leucina $(\mu \mathrm{mol} / \mathrm{L})$ & Isoleucina $(\mu \mathrm{mol} / \mathrm{L})$ & $\begin{array}{l}\text { Valina } \\
(\mu \mathrm{mol} / \mathrm{L})\end{array}$ \\
\hline 0 & & & 589 \\
$\mathrm{I}$ & 1.498 & 180 & 488 \\
2 & 1124 & 48 & 480 \\
3 & 877 & 32 & 230 \\
4 & 501 & 35 & 348 \\
5 & 623 & 35 & 269 \\
6 & 410 & 135 & 81 \\
7 & 380 & 158 & 189 \\
8 & 218 & 345 & 89 \\
\hline
\end{tabular}

precocemente e il suo decorso è caratterizzato da vari episodi di scompenso metabolico, che si sviluppano in condizioni d'ipercatabolismo.

Presentiamo il caso clinico di una crisi metabolica acuta in un ragazzo di 14 anni affetto da leucinosi, trattata con emofiltrazione veno-venosa continua (CVVH).

\section{Caso clinico}

Filippo, paziente di 14 anni, affetto da MSUD, si ricovera presso l'Unità Ospedaliera (U.O.) di Pediatria per la comparsa di malessere generale, stato confusionale e vomito. Dall'anamnesi si evince che ha trasgredito il regime dietetico e gli esami ematochimici mostrano alte concentrazioni plasmatiche di leucina (1235 micromol/L - v.n. 101 - 158); si pone diagnosi di crisi metabolica acuta in paziente affetto da leucinosi.

Già in passato F. aveva sviluppato diversi episodi di scompenso: il primo alla nascita, trattato con nutrizione enterale priva di leucina e 66 ore di CVVH, più altri 2 episodi all'età di 3 anni, insorti in seguito a febbre e faringotonsilliti, trattati entrambi con terapia nutrizionale.

Viene ricoverato presso l'U.O. di Pediatria e prontamente trattato con posizionamento di sondino naso-gastrico e nutrizione enterale priva di leucina con miscela di maltodestrine, più infusione di soluzione glucosata al $10 \%$. Nei giorni successivi si è assistito a un lento miglioramento delle condizioni generali e delle concentrazioni plasmatiche di leucina $(190,6 \mathrm{micromol} / \mathrm{L})$, ma la comparsa di febbre e vomito, di probabile natura virale, determina un nuovo peggioramento del quadro clinico. Di conseguenza viene sostituita la nutrizione enterale con l'infusione endovena di lipidi e di soluzione glucosata al $10 \%$. Ciononostante, le condizioni cliniche generali di F. continuano a peggiorare, il sensorio risulta compromesso (GCS 6) e i livelli di leucina aumentano nuovamente (1448,5 micromol/L), mentre l'EGA, gli indici di funzionalità renale ed epatica, l'emocromo e gli indici di flogosi risultano tutti nella norma. Quindi si rende necessario trasferire il ragazzo in Terapia Intensiva e, su indicazione del nefrologo, avviare in urgenza un trattamento di CVVH (tabella 1).

Per tutta la durata del trattamento F. è rimasto in respiro spontaneo, sostenuto con ossigenoterapia non invasiva; emodinamicamente stabile senza la necessità di amine. Durante la CRRT i livelli plasmatici dei BCAA sono stati monitorati due volte al giorno, insieme al controllo di ematocrito, proteine totali, indici di funzionalità renale, 


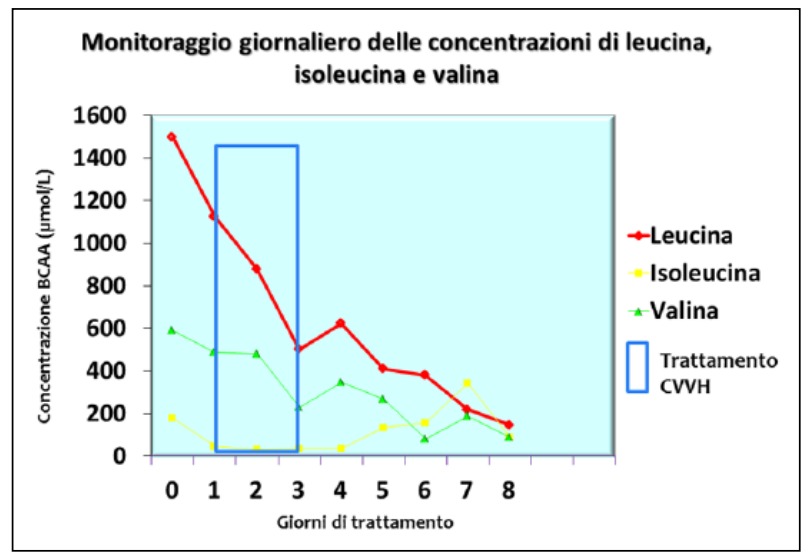

Figura I. II riquadro in blu comprende le concentrazioni plasmatiche dei BCAA al termine delle prime 24 ore di CVVH (giorno I), dopo 48 ore di trattamento (giorno 2) e dopo 72 ore (giorno 3).

elettroliti ed EGA. Nel contempo è stata continuata la nutrizione enterale ipercalorica.

Al termine del trattamento $\mathrm{F}$. ha recuperato l'integrità del sensorio (GCS 14) e le concentrazioni plasmatiche di leucina si sono notevolmente ridotte (fig. 1 e tabella 2); ha continuato la terapia nutrizionale per altri cinque giorni. L'episodio di scompenso non ha determinato sequele neurologiche, permettendo oggi al paziente di essere inserito in lista d'attesa per il trapianto di fegato.

\section{Discussione}

La malattia si presenta classicamente entro 48 ore dalla nascita ma in alcuni casi può esordire più tardivamente. $\mathrm{La}$ sintomatologia clinica è caratterizzata da: anoressia e vomito, ritardo psico-motorio, irritabilità, atassia, distonia, convulsioni e coma. ${ }^{2}$ Le urine presentano un odore caratteristico simile a quello dello sciroppo d'acero, derivante da un metabolita dell'isoleucina. Ė l'accumulo di leucina il principale responsabile del quadro clinico, poiché altera la regolazione del volume cellulare con riduzione della sodiemia, aumento dell'acqua intracellulare e conseguente edema cerebrale. Altresì la neurotossicità potrebbe dipendere dal rapido trasporto della leucina attraverso la barriera ematoencefalica, con aumentata produzione di glutammato, glutammina e GABA. ${ }^{2}$

Il gold standard per la diagnosi di MSUD è il dosaggio delle concentrazioni plasmatiche degli aminoacidi a catena ramificata (leucina, isoleucina, valina e allo-isoleucina), che risultano caratteristicamente elevati. Nelle urine si rilevano elevati livelli di chetoacidi a catena ramificata, lattato e piruvato.

Nel corso della sua vita il bambino affetto da leucinosi può sviluppare scompensi metabolici acuti, che possono manifestarsi con turbe del sensorio, e che sono potenzialmente associati ad un alto rischio di edema cerebrale e coma, o addirittura possono risultare fatali. Le crisi metaboliche insorgono in condizioni d'ipercatabolismo come errori alimentari, insufficiente intake di calorie o aminoacidi (anoressia e digiuno), traumi e infezioni. ${ }^{3}$

La clearance renale dei BCAA e dei loro derivati è scarsa, poiché essi vengono avidamente riassorbiti nel tubulo renale, e non è sufficiente per fronteggiare il loro accumulo, caratteristico delle fasi di scompenso. Queste ultime, però, richiedono un rapido abbattimento dei livelli plasmatici dei BCAA, poiché l'outcome neurologico (valutato anche in termini di QI) è tanto migliore quanto più rapidamente vengono rimossi dal circolo i metaboliti neurotossici. ${ }^{4}$ Infatti alcuni rapporti sostengono che la demielinizzazione cronica evidenziata mediante tomografia computerizzata (TC) o risonanza magnetica (RM) correla con i livelli plasmatici di leucina. ${ }^{5}$

La terapia delle crisi metaboliche è mirata a:

- sopprimere il catabolismo e facilitare l'utilizzo degli aminoacidi in eccesso per la sintesi proteica; ciò si ottiene mediante l'infusione di soluzione elettrolitica bilanciata e soluzione glucosata ipertonica, più la nutrizione parenterale $\mathrm{o}$ enterale priva di BCAA;

- rimuovere i metaboliti tossici accumulati mediante metodiche depurative extracorporee quali: IHD (emodialisi intermittente), CVVH (emofiltrazione veno-venosa continua), CVVHDF (emodiafiltrazione veno-venosa continua), dialisi peritoneale o exanguinotrasfusione. ${ }^{4}$

La dialisi peritoneale risulta efficace nella riduzione dei livelli di BCAA in neonati affetti da MSUD. La clearance dei BCAA è influenzata da diverse variabili: volume del dialisato, frequenza degli scambi e proprietà intrinseche del peritoneo. In bambini in condizioni cliniche severamente compromesse è difficile impiegare elevati volumi di dialisato e scambi frequenti, necessari per rimuovere più rapidamente i BCAA e ridurre la neurotossicità. ${ }^{6}$ Tuttavia la dialisi peritoneale, se eseguita da personale qualificato, secondo regole e procedure standardizzate, può essere considerata una procedura salvavita per i neonati con crisi metaboliche, specialmente nei paesi in via di sviluppo, che non dispongono facilmente delle tecniche extracorporee.

Col passare del tempo la terapia renale sostitutiva continua (CRRT) o intermittente (IHD) è stata sempre più impiegata, grazie all'evoluzione delle tecniche che oggi assicurano una migliore stabilità emodinamica.

L'IHD consente di ridurre le concentrazioni plasmatiche dei BCAA più rapidamente delle metodiche di CRRT. Poiché la leucina è una molecola a basso PM (131,2 Da), l'IHD ne determina un maggiore gradiente diffusivo tra il sangue del paziente e il dialisato. ${ }^{7}$ Ciò comporta un'esposizione più breve alla terapia anticoagulante. Tuttavia al termine dell'IHD può verificarsi il rebound delle concentrazioni plasmatiche di leucina per riequilibrio tra i compartimenti plasmatico e intracellulare, mentre la 
CRRT può teoricamente prevenire tale fenomeno per la maggiore durata del trattamento. ${ }^{8}$

Un altro potenziale svantaggio dell'IHD è rappresentato dal fatto che essa può compromettere l'emodinamica degli infanti. Ciò è particolarmente importante in pazienti a rischio di sviluppare edema cerebrale durante le crisi di scompenso, nei quali le riduzioni di pressione arteriosa e flusso ematico cerebrale potrebbero esacerbare le complicanze neurologiche. ${ }^{8}$

La CRRT, basandosi principalmente su forze convettive, determina una clearance dei BCAA più lenta rispetto all'IHD, ma parimenti efficace. La CVVHD riduce i livelli dei metaboliti tossici più rapidamente se comparata alla dialisi peritoneale, e offre il vantaggio di una maggiore stabilità emodinamica in neonati che non potrebbero tollerare un'ultrafiltrazione aggressiva. ${ }^{9}$ Ma le metodiche continue necessitano dell'infusione di terapia anticoagulante per tutta la durata del trattamento.

Anche la CVVH ad alti volumi ( $>35 \mathrm{ml} / \mathrm{kg} / \mathrm{h}$ ) consente una buona clearance dei BCAA. Tuttavia sia la dialisi peritoneale che l'IHD e la CRRT non sono scevre da complicanze. La prima può complicarsi con ostruzione del catetere e/o leakage dall'exit-site, la cui incidenza si è ridotta con l'utilizzo del catetere di Tenckhoff; l'IHD e la CRRT comportano un elevato rischio di trombosi ed emorragie.

Il trapianto di fegato rappresenta un'altra opzione terapeutica per pazienti affetti dalla forma classica di MSUD. Dopo il trapianto l'attività del complesso enzimatico BCKDC è equiparabile alla forma intermedia di MSUD e i pazienti non necessitano più delle restrizioni dietetiche, divenendo così protetti da ulteriori danni cerebrali.

La scelta della modalità dialitica più idonea è influenzata da diversi fattori, quali gli obiettivi terapeutici, i vantaggi e gli svantaggi di ciascuna metodica, le competenze del personale e le risorse disponibili.

L'exanguinotrasfusione, detta anche trasfusione sostitutiva, consiste nella rimozione di un volume ematico variabile da 5 a $20 \mathrm{ml}$ per ciclo, rimpiazzato dalla medesima quota di sangue o plasma fresco del donatore, che consente di ottenere il ricambio ematico del ricevente; è stata inizialmente impiegata per migliorare la clearance dei BCAA, sebbene solo transitoriamente..$^{10}$ Infatti, i livelli degli aminoacidi a catena ramificata vengono drasticamente ridotti ma rimbalzano ai livelli pretrattamento entro poche ore dal termine della procedura, per riequilibrio delle concentrazioni dai tessuti al sangue, richiedendo quindi la necessità di ripetere la metodica più volte.

Nel nostro paziente la scelta di una metodica continua in luogo di una intermittente è stata dettata dal timore di possibile insorgenza di instabilità emodinamica che potesse incrementare il rischio di danno neurologico ed eventuali sequele. Il rapido decremento dei livelli di leucina e outcome neurologico suggerisce che la CVVH è una valida opzione di trattamento dello scompenso metabolico acuto in pazienti affetti da MSUD.

\section{Dichiarazione di assenza di conflitto di interessi}

Gli Autori dichiarano di non avere conflitti di interessi.

\section{Finanziamenti}

Gli Autori dichiarano di non aver ricevuto finanziamenti specifici da qualsiasi ente nei settori pubblico, privato o senza fini di lucro.

\section{Bibliografia}

1. Chuang DT. Maple syrup urine disease: it has come a long way. J Pediatr 1998; 132: S17.

2. Korein J, Sansaricq C, Kalmijn M, et al. Maple syrup urine disease: clinical, EEG, and plasma amino acid correlations with a theoretical mechanism of acute neurotoxicity. Int $J$ Neurosci 1994; 79: 21.

3. Strauss KA, Wardley B, Robinson D, et al. Classical maple syrup urine disease and brain development: principles of management and formula design. Mol Genet Metab 2010; 99: 333-345.

4. Yoshino $\mathrm{M}^{1}$, Aoki $\mathrm{K}$, Akeda $\mathrm{H}$, et al. Management of acute metabolic decompensation in maple syrup urine disease: a multi-center study. Pediatr Int 1999; 41: 132-137.

5. Brismar J, Aqueel A, Brismar G, et al. Maple syrup urine disease: findings on CT and MR scans of the brain in 10 infants. Am J Neuroradiol 1990; 11: 1219-1228.

6. Puliyanda DP, Harmon WE, Peterschmitt MJ, et al. Utility of hemodialysis in maple syrup urine disease. Pediatr Nephrol 2002; 17: 239-242.

7. Gouyon JB, Desgrea J e Mousson C. Removal of branchedchain amino acids by peritoneal dialysis, continuous arteriovenous hemofiltration, and continuous arteriovenous hemodialysis in rabbits: implications for maple syrup urine disease treatment. Pediatr Res 1994; 35: 357-361.

8. Phan V, Clermont MJ, Merouani A, et al. Duration of extracorporeal therapy in acute maple syrup urine disease: a kinetic model. PediatrNephrol 2006; 21: 698-704.

9. Jouvet P, Poggi F, Rabier D, et al. Continuous venovenous hemodiafiltration in the acute phase of neonatal maple syrup urine disease. J Inher Metab Dis 1997; 20: 463-472.

10. Wendel U, Langenbeck U, Lombeck I, et al. Exchange transfusion in acute episodes of Maple Syrup Urine Disease - studies on branched-chain amino and keto acids. Eur $J$ Pediatr 1982; 138: 293-296. 